\title{
Production of HlyA and ClyA haemolysins among quinolone-resistant Escherichia coli isolated from clinical samples
}

\author{
Alicia Márquez-López ${ }^{1 *}$, Belén Ruiz del Castillo ${ }^{1}$, María Eliecer Cano ${ }^{1}$, Cristina Rodríguez-Mirones ${ }^{1}$, Jesús Oteo ${ }^{3}$, \\ David Sáez ${ }^{3}$ and Luis Martínez-Martínez ${ }^{1,2}$
}

\begin{abstract}
Most Escherichia coli resistant to quinolones are not haemolytic. The objective of this study was to determine the phylogroup, clonal relationship, mechanism of quinolone resistance and virulence factors in 70 haemolytic E. coli resistant to nalidixic acid. Sixty-six isolates contained the hlyA gene, belonged to phylogroup B2, and 61 of them presented low-level resistance to fluoroquinolones. Four isolates presented high-level resistance to fluoroquinolones, contained the clyA gene and were included in phylogroup D. One single isolate (phylogroup D, with low level resistance to fluoroquinolones) contained both cytotoxins.
\end{abstract}

Keywords: Haemolysis, Escherichia coli, Quinolone resistance, hlyA, clyA

Escherichia coli strains causing extraintestinal infections (ExPEC) express different virulence factors (VF), including $\alpha$-haemolysin (HlyA) and cytolysin A (ClyA, SheA or HlyE), both of which can induce osmotic lysis of erythrocytes (Kerényi et al. 2005). However, quinolone-resistant clinical isolates of $E$. coli ExPEC are frequently non-haemolytic (Horcajada et al. 2005; Martínez-Martínez et al. 1999). This may be related to the observation that strains of phylogroup B2 and D usually contain more VF (including haemolysins) than those of phylogroups $\mathrm{A}$ and B1 (Clermont et al. 2000; Houdouin et al. 2007). On the other hand, isolates of groups A and B1 are more frequently resistant to quinolones than those of phylogroup B2 and D (with the notable exception of some particular multiresistant clones, such as the O25:H4-B2-ST131 clone) (Houdouin et al. 2007; Takahashi et al. 2009). Thus, a detailed analysis of clinical isolates presenting the unusual association of quinolone resistance and haemolysis production may be helpful to understand the relationship between virulence and resistance in E. coli.

\footnotetext{
*Correspondence: alicia_marquez@hotmail.com

${ }^{1}$ Service of Microbiology, University Hospital Marqués de Valdecilla-IFIMAV, Santander, Spain

Full list of author information is available at the end of the article
}

From November 2002 to March 2010, 33042 Escherichia coli were isolated from clinical samples in our laboratory (University Hospital Marqués de Valdecilla, Santander, Spain). Seventy (0.2\% of all isolates) were haemolytic and resistant to nalidixic acid. Organisms were cultured from urine (63 isolates), blood (2), soft tissue abscess (1), abdominal abscess (1), wound (1), tracheal aspirate (1) or skin ulcer (1). Bacteria were identified with the Microscan WalkAway 96 system (Dade Behring, CA, USA). MICs of nalidixic acid (SigmaAldrich, Madrid, Spain), ciprofloxacin (Sigma-Aldrich), norfloxacin (Sigma-Aldrich) and levofloxacin (Aventis Pharma, Madrid, Spain) were determined by broth microdilution, according to CLSI guidelines (Clinical and Laboratory Standards Institute 2011). Haemolysin production was assessed in sheep blood agar (Oxoid, Madrid, Spain). An organism was considered haemolytic when a clear halo was observed around isolated colonies after overnight incubation at $37^{\circ} \mathrm{C}$. The phylogroup was determined by multiplex PCR (Clermont et al. 2000). Clonal relationship was evaluated by repetitive extragenic palindromic PCR (REP-PCR); isolates were considered unrelated if more than two bands of difference were observed. Additionally, 27 isolates representative of the different patterns obtained by REP-PCR (1-4 isolates per REP-PCR pattern), were 
typed by Pulsed-Field Gel Electrophoresis (PFGE) and analyzed by Multilocus Sequence Typing (MLST) according to the protocol specified at the E. coli MLST website (http://mlst.ucc.ie/mlst/dbs/Ecoli). This resulted in the recognition of 13 REP-PCR pattterns, 14 PFGE patterns and 11 sequence types (ST), which combined allowed to define 15 distinct organisms. The quinolone resistance-determining regions (QRDRs) of gyrA and $\operatorname{parC}$ were amplified (Vila et al. 1996a; Vila et al. 1996b) and sequenced in the Molecular Genetics Unit of the HUMV. The presence of genes coding for five major horizontally transmissible quinolone resistance determinants (qnrA, qnrB, qurS, qepA, and aac-(6I)-Ib-cr) was investigated by multiplex PCR (Cano et al. 2009). The presence of the $h l y A$ and $c l y A$ genes was evaluated by PCR (Kerényi et al. 2005). Additionally, 19 virulence-associated genes (traT, iutA, bmaE, iroN, sfaS, afa/dra, ibeA, fyuA, fimH, Pai, K1, K5, KpsMTII, KpsMTIII, cnf1, focG, gafD, papC, sat) were analyzed in the previously indicated 15 isolates (Johnson \& Stell 2000; Bonacorsi et al. 2003). The VF score of each isolate was calculated as the number of VF genes for which the isolate tested positive (Bert et al. 2008).

The distribution of MICs of the four evaluated quinolones is presented in Additional file 1. According to the CLSI breakpoints, 65 (92.8\%), 64 (91.4\%) and 66 $(94.3 \%)$ out of the 70 isolates were susceptible to ciprofloxacin, norfloxacin and levofloxacin, respectively. When the breakpoints defined by EUCAST were considered, the percentages of susceptibility to ciprofloxacin, norfloxacin and levofloxacin were $88.6 \%, 54.3 \%$ and $91.4 \%$, respectively. All isolates contained one or two mutations in $g y r A$ alone or (in six cases) associated to a mutation in parC (Table 1). The most common change in GyrA was serine to leucine in position 83 . Plasmid-mediated quinolone resistance determinants were not detected. Interestingly, although all our haemolytic isolates contained one or more DNA-gyrase mutations, most of them are defined as susceptible to fluoroquinolones according to the CLSI breakpoints (Clinical and Laboratory Standards Institute 2011), or even to the more restrictive breakpoints defined by EUCAST (European Committee on Antimicrobial Susceptibility Testing 2012).

Sixty-six (94.3\%) and five (7.2\%) isolates corresponded to phylogroups B2 and D. Most isolates of phylogroup B2 belonged to ST73, ST12, ST372 or ST131, while the majority of the isolates of the group D belonged to ST350 (Table 1). Molecular typing has shown a certain degree of clonal diversity among the isolates we have evaluated, with some clones containing single isolates and some other including multiple isolates. According to these data, a qualitative analysis indicates that our isolates do not represent the dissemination of only one single or just a few clones.

Sixty-five (92.9\%) isolates contained the $h l y A$ gene, four $(5.7 \%)$ isolates contained the $\operatorname{cly} A$ gene and one (1.4\%) single isolate contained both hlyA and clyA. In a previous study by Kerényi et al. on 540 extraintestinal $E$. coli strains, the clyA gene was identified in 241 isolates and hlyA in 198 isolates (Kerényi et al. 2005); these authors did not find an isolate simultaneously containing both haemolysin genes and suggested their possible incompatibility, which is in contrast with our observation of an isolate with the two haemolysin genes. MICs of ciprofloxacin for 61 out of $65(93.8 \%)$ isolates with only hlyA were $\leq 0.5 \mu \mathrm{g} /$ $\mathrm{ml}$ while MICs of this agent against the four isolates containing only clyA were $\geq 8 \mu \mathrm{g} / \mathrm{ml}$. The MICs of the single isolate with both haemolysins was $0.125 \mu \mathrm{g} / \mathrm{ml}$. It is difficult to determine if the presence of clyA alone is more common in isolates with high level fluoroquinolone resistance, as we have found this association in just a small number of isolates (all of them of ST 350). It would be possible that this relationship is related to the phylogenetic background of the isolates, as hlyA was linked to phylogroup B2, while clyA was linked to phylogroup D. Additional studies are in progress to evaluate this observation in a large collection of E. coli consecutively isolated from bacteremic episodes. This analysis would also provide more data on the presence of $h l y A$ in strains with just low-level resistance to fluoroquinolones.

The VF score was slightly higher for isolates of phylogroup B2 (10.9) than for those of phylogroup D (7.6). In the 15 isolates studied in detail, the most frequent virulence-associated genes (Table 1) were fimH (100\%), cnf1 (87\%), traT (80\%), fyuA (80\%) and papC (80\%). None of the isolates presented kpsMTIII. Other genes such as PAI (73\%), iroN (73\%), K5 (67\%), kpsMTIII (67\%), ibeA (53\%), iutA (46.7\%), sfaS (33.3\%), focG (26.6\%), afa/dra (20\%), sat (13.3\%), bmaE (6.6\%), $K 1$ (6.6\%) and gafD (6.6\%) were less frequently detected. Isolates with $c l y A$ also contained more frequently iutA and ibeA. Previous studies have shown that the fimH and traT genes are more frequently found in E. coli isolates (irrespective of their susceptibility to quinolones) than other virulence genes such cnf1 or hlyA (Takahashi et al. 2009; Cooke et al. 2010). The prevalence of cnfl in this study, which is higher than that previously described in isolates causing urinary and skin infections could be explained by the association of $c n f 1$ and $h l y A$ in a pathogenicity island, as previously reported (Johnson \& Stell 2000; Petkovsek et al. 2009; Schmidt \& Hensel 2004). 
Table 1 Phylogroup (PhG), clonal relationship defined by REP-PCR patterns (REP), Pulsed-Field Gel Electrophoresis (PFGE), and Multilocus Sequence Typing (MLST), MIC of quinolones, mutations in topoisomerase genes, and presence of haemolysins and virulence-associated genes in a representative set of isolates

\begin{tabular}{|c|c|c|c|c|c|c|c|c|c|c|c|c|c|c|c|c|c|c|c|c|c|c|}
\hline \multirow[b]{2}{*}{ Isolate } & \multirow[b]{2}{*}{ PhG } & \multirow[b]{2}{*}{$\operatorname{REP}(n)$} & \multirow[b]{2}{*}{ PFGE } & \multicolumn{2}{|c|}{ MLST } & \multicolumn{4}{|c|}{ MIC $(\mu \mathrm{g} / \mathrm{ml})$} & \multicolumn{2}{|c|}{ Mutations } & \multicolumn{2}{|c|}{ Haemolysins } & \multicolumn{9}{|c|}{ Virulence-associated genes } \\
\hline & & & & ST & ST cplx & NAL & NOR & CIP & LEV & gyrA & parC & hlyA & CIYA & trat & iroN & $i b e A$ & fyuA & cnf1 & PAl & papC & K5 & KpsMT \\
\hline Hly-557 & $\mathrm{D}$ & A (4) & $1 \mathrm{~B}$ & 350 & 350 & $>512$ & $>16$ & 16 & 8 & S83L D87N & $\mathrm{S} 80 \mathrm{l}$ & - & + & - & - & + & - & - & - & - & - & - \\
\hline Hly-1872 & D & & 2 & 57 & & $>512$ & $>16$ & 8 & 4 & S83L D87N & S80l & - & + & + & - & + & - & - & - & - & - & - \\
\hline Hly-263 & D & $B(1)$ & 3 & 405 & 405 & 32 & 0.5 & 0.125 & 0.125 & D87G & / & + & + & + & - & + & + & + & + & + & + & + \\
\hline Hly-520 & B2 & $C(16)$ & $4 \mathrm{~A}$ & 12 & 12 & 256 & 0.5 & 0.25 & 0.25 & S83L & / & + & - & + & + & - & + & + & + & + & + & + \\
\hline Hly-6129 & B2 & & 5 & & & 256 & 1 & 0.25 & 0.25 & S83L & I & + & - & - & + & - & + & + & + & + & - & - \\
\hline Hly-2304 & B2 & $\mathrm{D}(1)$ & 6 & 599 & 12 & $>512$ & $>16$ & 1 & 1 & S83L & / & + & - & + & - & - & + & + & - & + & - & - \\
\hline Hly-4105 & B2 & E (19) & 7 & 372 & None & 128 & 0.5 & 0.25 & 1 & S83L & / & + & - & - & + & + & + & + & + & + & - & - \\
\hline Hly-2425 & B2 & $F(7)$ & $8 \mathrm{~A}$ & 372 & None & 128 & 1 & 0.25 & 0.25 & S83L & I & + & - & + & + & + & + & + & + & + & + & + \\
\hline Hly-4530 & B2 & G (10) & 9 & 73 & 73 & 128 & 0.5 & 0.25 & 0.25 & S83L & I & + & - & + & + & - & + & + & + & + & + & + \\
\hline Hly-3308 & B2 & H (19) & $10 C$ & 73 & 73 & 64 & 0.5 & 0.125 & 0.125 & D87Y & I & + & - & + & + & - & + & + & + & - & + & + \\
\hline Hly-5126 & B2 & I (2) & 11 & 127 & None & 64 & 1 & 0.25 & 0.5 & D82N & I & + & - & + & + & - & + & + & + & + & + & + \\
\hline Hly-3606 & B2 & $J(1)$ & 12 & 127 & None & 64 & 0.5 & 0.25 & 0.25 & S83L & / & + & - & + & + & - & + & + & + & + & + & + \\
\hline Hly-2605 & B2 & K (6) & $13 C$ & 131 & None & 256 & 1 & 0.25 & 0.5 & S83L & I & + & - & + & + & + & + & + & + & + & + & + \\
\hline Hly-3438 & B2 & $L(1)$ & $13 \mathrm{D}$ & & & 128 & 0.5 & 0.25 & 0.25 & S83L & I & + & - & + & + & + & + & + & + & + & + & + \\
\hline Hly-1248 & B2 & $M(1)$ & 14 & 537 & 14 & $>512$ & 4 & 1 & 0.5 & S83L & S80R & + & - & + & + & + & - & + & - & + & + & + \\
\hline
\end{tabular}

ST = Sequence Typing, ST cplx = Sequence Typing complexes, NAL = nalidixic acid, NOR = norfloxacin, CIP = ciprofloxacin, LEV = levoflofacin, $S=$ Serine, L = Leucine, D = Aspartic acid, N= Asparagine, I = isoleucine, $\mathrm{G}=$ glycine, $\mathrm{Y}=$ tyrosine and $\mathrm{R}=$ arginine. 


\section{Additional file 1}

Additional file 1: Table 1. Distribution of absolute (and cumulative) MICs $(\mu \mathrm{g} / \mathrm{ml})$ of four quinolones against 70 haemolytic E. coli isolates. Values corresponding to resistance and intermediate susceptibility (according to CLSI criteria) are shadowed in dark and pale grey, respectively.

\section{Competing interest}

The authors declare that they have no competing interest.

\section{Authors' contributions}

LMM conceived the study, contributed to designing the experiments and participated in drafting the manuscript. AML, BRC, MEC and CRM performed different experiments. JO and DS carried out the studies in virulenceassociated genes detection. LMM, AML and JO wrote the manuscript. All authors read and approved the final manuscript.

\section{Acknowledgements}

Supported by Ministerio de Economía y Competitividad, Instituto de Salud Carlos III, Madrid, Spain, co-financed by European Development Regional Fund "A way to achieve Europe" ERDF, Spanish Network for the Research in Infectious Diseases (REIPI RD12/0015). Alicia Márquez-López was supported by the REIPI and has been supported by a grant from the Instituto de Formación e Investigación Marqués de Valdecilla (IFIMAV), Santander, Spain. We want to thank Eduardo López for his review of English version of the manuscript.

\section{Author details}

${ }^{1}$ Service of Microbiology, University Hospital Marqués de Valdecilla-IFIMAV Santander, Spain. ${ }^{2}$ Departament of Molecular Biology, University of Cantabria, Santander, Spain. ${ }^{3}$ Antibiotic Laboratory, Bacteriology Service, Centro Nacional de Microbiología, Instituto de Salud Carlos III, Madrid, Spain.

Received: 24 January 2013 Accepted: 15 February 2013

Published: 27 February 2013

\section{References}

Bert F, Panhard X, Johnson J, Lecuyer H, Moreau R, Le Grand J et al (2008) Genetic background of Escherichia coli isolates from patients with spontaneous bacterial peritonitis: relationship with host factors and prognosis. Clin Microbiol Infect 14:1034-1040

Bonacorsi S, Clermont O, Houdouin V, Cordevant C, Brahimi N, Marecat A et al (2003) Molecular analysis and experimental virulence of French and North American Escherichia coli neonatal meningitis isolates: identification of a new virulent clone. J Infect Dis 187:1895-1906

Cano ME, Rodríguez-Martinez JM, Agüero J, Pascual A, Calvo J, García-Lobo JM et al (2009) Detection of plasmid-mediated quinolone resistance genes in clinical isolates of Enterobacter spp. In spain. J Clin Microbiol 47:2033-2039

Clermont O, Bonacorsi S, Bingen E (2000) Rapid and simple determination of the Escherichia coli phylogenetic group. Appl Environ Microbiol 66:4555-4558

Clinical and Laboratory Standards Institute (2011) Performance Standards for Antimicrobial Susceptibility Testing M100-S21. CLSI, Wayne, Pa

Cooke NM, Smith SG, Kelleher M, Rogers TR (2010) Major differences exist in frequencies of virulence factors and multidrug resistance between community and nosocomial Escherichia coli bloodstream isolates. J Clin Microbiol 48:1099-1104

European Committee on Antimicrobial Susceptibility Testing (2012) Clinical Breakpoint Table v.2.0. EUCAST, Available: http://www.eucast.org/fileadmin/ src/media/PDFs/EUCAST_files/Breakpoint_tables/Breakpoint_table_v_2.0_ 120221.pdf

Horcajada JP, Soto S, Gajewski A, Smithson A, Jímenez de Anta MT, Mensa J et al (2005) Quinolone-resistant uropathogenic Escherichia coli strains from phylogenetic group B2 have fewer virulence factors than their susceptible counterparts. J Clin Microbiol 43:2962-2964

Houdouin V, Bonacorsi S, Mahjoub-Messai F, Mariani-Kurkdjian P, Bidet P, Sebag $\mathrm{G}$ et al (2007) Phylogenetic groups and virulence factors of Escherichia coli strains causing pyelonephritis in children with and without urinary tract abnormalities. Clin Microbiol Infect 13:740-742
Johnson JR, Stell AL (2000) Extended virulence genotypes of Escherichia coli strains from patients with urosepsis in relation to phylogeny and host compromise. J Infect Dis 181:261-272

Kerényi M, Allison HE, Bátai I, Sonnevend Á, Emödy L, Plaveczky N et al (2005) Occurrence of hlyA and sheA genes in Extraintestinal Escherichia coli strains. J Clin Microbiol 43:2965-2968

Martínez-Martínez L, Fernández F, Perea EJ (1999) Relationship between haemolysis production and resistance to fluoroquinolones among clinical isolates of Escherichia coli. J Antimicrob Chemother 43:277-279

Petkovsek Z, Elersic K, Gubina M, Zqur-Bertok D, Starcic EM (2009) Virulence potential of Escherichia coli isolates from skin and soft tissue infections. J Clin Microbiol 47:1811-1817

Schmidt H, Hensel M (2004) Pathogenicity islands in 363 bacterial pathogenesis. Clin Microbiol Rev 17:14-56

Takahashi A, Muratani T, Yasuda M, Takahashi S, Monde K, Ishikawa K et al (2009) Genetic profiles of fluoroquinolone-resistant Escherichia coli isolates obtained from patients with cystitis: phylogeny, virulence factors, PAlusp subtypes, and mutation patterns. J Clin Microbiol 47:791-795

Vila J, Ruiz J, Goñi P, Marcos A, Jimenez de Anta MT (1996a) Mutation in gyrA gene of quinolone-resistant clinical isolates of Acinetobacter baumannii. Antimicrob Agents Chemother 39:1201-1203

Vila J, Ruiz J, Goñi P, Jimenez de Anta MT (1996b) Detection of mutations in parC in Quinolone-resistant clinical isolates of Escherichia coli. Antimicrob Agents Chemother 40:491-493

doi:10.1186/2193-1801-2-71

Cite this article as: Márquez-López et al:: Production of HlyA and ClyA haemolysins among quinolone-resistant Escherichia coli isolated from clinical samples. SpringerPlus 2013 2:71.

\section{Submit your manuscript to a SpringerOpen ${ }^{\odot}$ journal and benefit from:}

- Convenient online submission

- Rigorous peer review

- Immediate publication on acceptance

- Open access: articles freely available online

- High visibility within the field

- Retaining the copyright to your article

Submit your next manuscript at $>$ springeropen.com 